EPJ manuscript No.

(will be inserted by the editor)

\title{
Atom interferometry gravity-gradiometer for the determination of the Newtonian gravitational constant G
}

\author{
A. Bertoldi, G. Lamporesi, L. Cacciapuoti ${ }^{\mathrm{a}}$, M. de Angelis ${ }^{\mathrm{b}}$, M. Fattori ${ }^{\mathrm{c}}$, T. Petelski ${ }^{\mathrm{d}}$, A. Peters $^{\mathrm{e}}$, M. Prevedelli $^{\mathrm{f}}$, \\ J. Stuhler ${ }^{c}$, and G. M. Tino ${ }^{\mathrm{g}}$ \\ ' Dipartimento di Fisica and LENS, Università di Firenze; INFN, Sezione di Firenze \\ Via Sansone 1, 50019 Sesto Fiorentino (Firenze), Italy \\ Received: date / Revised version: date

\begin{abstract}
We developed a gravity-gradiometer based on atom interferometry for the determination of the Newtonian gravitational constant $G$. The apparatus, combining a Rb fountain, Raman interferometry and a juggling scheme for fast launch of two atomic clouds, was specifically designed to reduce possible systematic effects. We present instrument performances and show that the sensor is able to detect the gravitational field induced by source masses. A discussion of projected accuracy for $G$ measurement using this new scheme shows that the results of the experiment will be significant to discriminate between previous inconsistent values.
\end{abstract}

PACS. 03.75.Dg Atom and neutron interferometry - 04.80.-y Experimental studies of gravity - 06.20.Jr Determination of fundamental constants

\section{Introduction}

Recent advances in atom interferometry led to the development of new methods for fundamental physics experiments and for applications [1. In particular, atom interferometers are new tools for experimental gravitation as, for example, for precision measurements of gravity acceleration 2, gravity gradients 3, equivalence principle tests 4], $1 / r^{2}$ law test [5] 6], and for possible applications in geophysics [2,8]. Ongoing studies show that future experiments in space [5] will allow to take full advantage of the potential sensitivity of atom interferometers using, for example, atom gyroscopes [9 10] to test general relativity predictions [11. The possibility of using atom interferometry for gravitational waves detection was also investigated 12, $13,14$.

In this paper, we describe an atom interferometer developed for a precise determination of the Newtonian gravitational constant $G$. The basic idea of the experiment and the planned precision were presented in [15]. Here we dis-

a European Space Agency, ESTEC, 2200 AG Nordwijk, NL

b On leave from: Istituto Cibernetica CNR,80078 Pozzuoli, I

c Present address: Physikalisches Institut, Universität Stuttgart, 70550 Stuttgart, D

d Present address: European Patent Office,80469 München,D

e Institut für Physik, Humboldt-Universität zu Berlin, 10117 Berlin, D

f Dipartimento di Chimica Fisica e Inorganica, Università di Bologna, 40136 Bologna, I

g E-mail: guglielmo.tino@fi.infn.it cuss the interferometer performances and show that the sensor is able to detect the gravitational field induced by source masses. The projected accuracy for $G$ measurement using this new scheme shows that the results of the experiment will be significant to discriminate between existing inconsistent values.

In fact, because of the importance of this fundamental physical constant, more than 300 experiments were performed to determine its value but the results are not in agreement. As a result, the present recommended CODATA value $\left(G=6.6742(10) \times 10^{-11} \mathrm{~m}^{3} \mathrm{~kg}^{-1} \mathrm{~s}^{-2}\right)$ is affected by an uncertainty of $150 \mathrm{ppm}$, which is much larger than for any other fundamental physical constant 16. With a few exceptions, most experiments were performed using conceptually similar schemes based on suspended macroscopic masses as probes and torsion balances or pendula as detectors.

In our experiment, freely falling atoms act as probes of the gravitational field and an atom interferometry scheme is used to measure the effect of nearby well-characterized source masses (Figure 1). ${ }^{87} \mathrm{Rb}$ atoms, trapped and cooled in a magneto-optical trap (MOT), are launched upwards in a vertical vacuum tube with a moving optical molasses scheme, producing an atomic fountain. Near the apogee of the atomic trajectory, a measurement of their vertical acceleration is performed by a Raman interferometry scheme 17. External source masses are positioned in two different configurations and the induced phase shift is measured as a function of masses positions. In order to suppress common-mode noise and to reduce systematic effects, a 


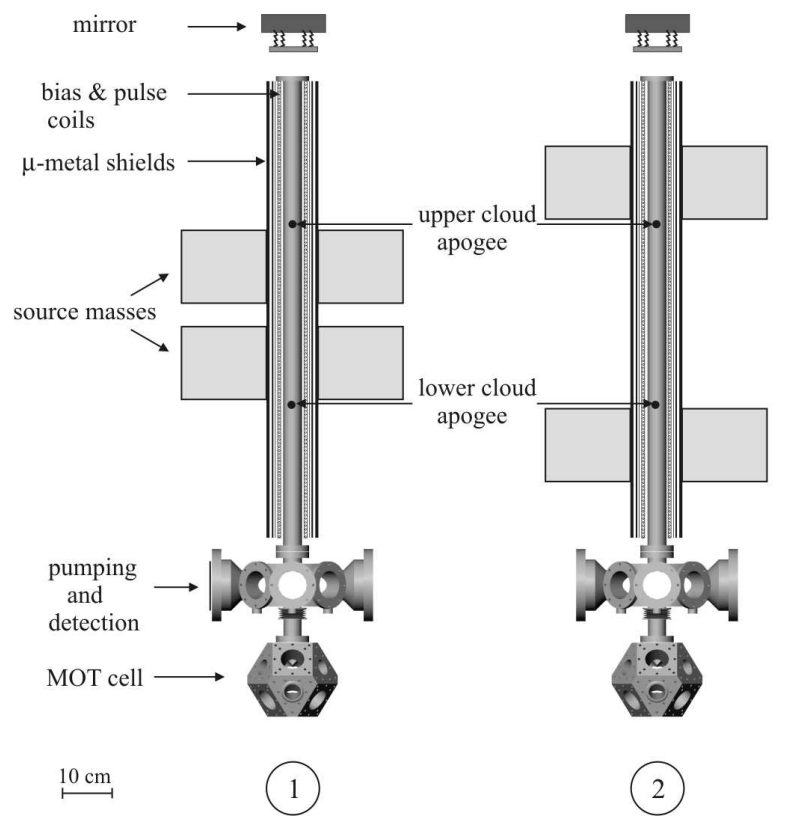

Figure 1. Scheme of the experimental apparatus showing the atomic fountain set-up and the two source masses configurations. The laser beams and optical system are not shown.

double-differential scheme has been adopted. The vertical acceleration is simultaneously measured in two vertically separated positions with two atomic samples, that are launched in rapid sequence with a juggling method. From the differential acceleration measurements as a function of the position of source masses, and from the knowledge of the mass distribution, the value of $G$ can be determined.

The paper is organized as follows: In Section 2 we introduce Raman interferometry and the basic idea of the experiment to measure $G$. Section 3 describes the apparatus and the experimental sequence. A characterization of interferometer and gradiometer performaces is presented in Section 4 In Section 5 we report the detection of external source masses. In Section [6] we discuss the expected performance with the final configuration and the projected accuracy in the measurement of $G$.

\section{Raman interferometry basics and idea of the experiment}

In this section, we discuss the basic idea of the experiment, the scheme of Raman interferometry and its application to measure $G$. A more detailed discussion can be found in [15] and references therein.

In a Raman interferometry-based gravimeter, atoms in an atomic fountain are illuminated by a sequence of three light pulses. The light pulses are realized with two laser beams, which have frequencies $\omega_{1}$ and $\omega_{2}$ close to transitions of a $\Lambda$-type three-level atom with two lower states $|a\rangle$ and $|b\rangle$ and an excited state $|e\rangle$. The laser beams propagate along the vertical $z$-axis in opposite directions and with wavevectors $\boldsymbol{k}_{1}=k_{1} \boldsymbol{e}_{z}$ and $\boldsymbol{k}_{2}=-k_{2} \boldsymbol{e}_{z}\left(k_{i}=\omega_{i} / c, \mathrm{i}=\right.$
1,2). The light pulses drive two-photon Raman transitions between $|a\rangle$ and $|b\rangle$. A $\pi$-pulse, which has a duration of $\tau=\pi / \Omega$ ( $\Omega$ being the two photon Rabi frequency), switches the atomic state from $|a\rangle$ to $|b\rangle$ or viceversa. A $\pi / 2$-pulse with duration $\tau=\pi /(2 \Omega)$ splits the atom wavefunction into an equal superposition of $|a\rangle$ and $|b\rangle$. Besides altering the real amplitudes $\alpha, \beta$ of the atomic wavefunction $\Psi=\alpha \mathrm{e}^{i \Phi_{\alpha}}|a\rangle+\beta \mathrm{e}^{i \Phi_{\beta}}|b\rangle$, the light field can also modify the atomic momentum and the phase. An atom that changes its internal state receives a momentum transfer by an amount of $\hbar k_{\text {eff }}=\hbar\left(k_{1}+k_{2}\right)$. At the same time, the phases $\Phi_{\alpha, \beta}$ are modified according to the local phase of the light field.

The interferometer is realized by a sequence of a $\pi / 2$ pulse, a $\pi$-pulse and $\pi / 2$-pulse, each separated by the time $T$, that produces two possible paths, I and II, in spacetime. The first $(\pi / 2$ pulse) splits the atomic wave packet, the second $(\pi$ pulse) induces the internal and external state inversion and the third $(\pi / 2$ pulse) recombines the matter waves after their different space-time evolution. After recombination of the two paths, at the output of the interferometer the probability of finding the atoms in state $|a\rangle$ shows a typical interference-like behaviour:

$$
N_{a} / N \propto 1-\cos \left(\Phi_{\mathrm{I}}-\Phi_{\mathrm{II}}\right)
$$

$\Phi_{\text {I,II }}$ are the phases accumulated on path I and II, respectively. The phases $\Phi_{\text {I }}$ and $\Phi_{\text {II }}$ depend on the local phase of the light field as seen by the atoms during Raman pulses. This links the vertical atomic position to the phase evolution of the laser field. The phase evolution depends on the effective frequency $\omega_{\text {eff }}(t)=\omega_{1}(t)-\omega_{2}(t)$ and on the phase relation between the Raman pulses. Usually, one varies the effective frequency linearly in time to compensate for the first order Doppler effect of the free-falling atoms and keep the Raman resonance condition (effective frequency matching the energy splitting of the two lower states). With $\omega_{\text {eff }}(t)=\omega_{\text {eff, } 0}-\beta t$ one then obtains:

$$
\Phi_{\mathrm{I}}-\Phi_{\mathrm{II}}=\left(\beta-k_{\mathrm{eff}} g\right) T^{2}-\phi(0)+2 \phi(T)-\phi(2 T)
$$

The compensation of the Doppler effect $\left(\beta=k_{\text {eff }} g\right)$ and an unperturbed evolution of the laser phase $\phi$ leads to $\Phi_{\mathrm{I}}-\Phi_{\mathrm{II}}=0$. Actively changing the laser phase between $T$ and $2 T$ by $\delta \phi$ will result in $\phi(2 T)=\phi(0)+\delta \phi=\phi(T)+\delta \phi$ and hence $\Phi_{\mathrm{I}}-\Phi_{\mathrm{II}}=-\delta \phi$. In this way, one can scan the interference fringe to prove $\left(\beta-k_{\text {eff }} g\right)=0$ (for right $\beta$ ) or reveal the phase offset $\left(\beta-k_{\text {eff }} g\right) T^{2}$ (for imperfect Doppler compensation). In both cases, the value of $g$ is obtained combining the measured phase offset and the value of $\beta$, which is set by a frequency generator.

For the measurement of $G$, we use a Raman interferometer to detect the change in atoms acceleration induced by external source masses. In order to achieve high sensitivity and accuracy, however, the experimental scheme was developed with important specific features.

First, we launch two clouds of atoms to realize two fountains that are displaced vertically (see Figure 1). The Raman pulses act on both clouds simultaneously and generate two interferometers at the same time. In a detection of the differential phase shift between the two interferometers, spatial homogeneous accelerations cancel and 
common mode measurement noise is reduced. The twocloud setup results in an atomic gravity gradiometer. If the trajectory of the first cloud is located above the source masses, atoms will experience an induced acceleration in $-z$ direction. In contrast, choosing the trajectory of the second cloud below the source masses, the induced acceleration of this cloud will be in $+z$ direction. Taking the difference of these two accelerations yields a signal, which is about twice the one obtained with only one cloud.

Second, we determine the differential interferometer phase shifts with the source masses at distinct positions (positions 1 and 2 in Figure 1). Evaluating the difference between the measurements further reduces systematic spurious effects if they are constant over the time scale of the source masses repositioning.

Third, the atomic trajectories and the shape and positioning of source masses were optimized to reduce experimental sensitivity to crucial parameters like precision and stability of the atomic fountain.

The combination of these features will allow to reach the targeted accuracy $\Delta G / G \approx 10^{-4}$.

\section{Experimental setup}

The experimental apparatus consists of the vacuum chamber, the laser sources and optical set up for the production of the double fountain of Rb atoms using a juggling procedure, the apparatus for Raman pulse interferometry, and the system of source masses, their support and precision positioning components. The experimental sequence, timing and data acquisition are computer controlled. In this section, we describe the main parts of the apparatus. More details can be found in 18 .

\subsection{Atomic fountain and juggling apparatus}

The fountain of ${ }^{87} \mathrm{Rb}$ atoms is produced using a magnetooptical trap and moving-molasses scheme. The laser beams are in a 1-1-1 six-beam $\sigma^{+} / \sigma^{-}$configuration. This keeps the central vertical axis free for the Raman laser beams and allows to realize a stable and precise atomic fountain.

The relevant parts of the vacuum system are shown in Figure 1 It consists of three main parts: The lowest part is a titanium cube with cut edges where atoms are trapped in the MOT from the vapour produced by a dispenser (SAES Getters 5G0807), cooled and launched in the fountain. The middle part of the vacuum system is a thermally demagnetized 316 LN stainless steel cell. This cell is used for pumping and to detect the atoms. The top part of the vacuum system is the interferometer tube. It is $1 \mathrm{~m}$ long, has a diameter of $40 \mathrm{~mm}$ and is made of titanium. The tube is magnetically shielded with two coaxial cylinders of $\mu$-metal (Amuneal) that are $0.76 \mathrm{~mm}$ thick and $1 \mathrm{~m}$ long, with internal diameters of $74 \mathrm{~mm}$ and $84 \mathrm{~mm}$. The attenuation of the radial and axial components of the external field is $76 \mathrm{~dB}$ and $69 \mathrm{~dB}$, respectively. The saturation field $\mathrm{B}_{\mathrm{S}}$ is about $6 \mathrm{mT}$.
A magnetic field gradient of $75 \mathrm{mT} / \mathrm{m}$ in the MOT region is generated by a water-cooled pair of coils in antiHelmholtz configuration. The cooling radiation is generated by a tapered amplifier delivering $500 \mathrm{~mW}$ output power. It is injected with $25 \mathrm{~mW}$ from a laser (New Focus Vortex $6000,65 \mathrm{~mW}$ at $125 \mathrm{~mA}$ ) frequency-stabilized on the $5^{2} \mathrm{~S}_{1 / 2}, \mathrm{~F}=2 \rightarrow 5^{2} \mathrm{P}_{3 / 2}, \mathrm{~F}=3$ transition of ${ }^{87} \mathrm{Rb}$. This laser also acts as the main frequency reference for the experiment. The radiation for the MOT is detuned by $3 \Gamma$ ( $\Gamma=2 \pi \cdot 6.1 \mathrm{MHz}$ ) to the red of the resonance.

Repumping light is provided by an extended cavity diode laser, frequency-locked to the reference laser and optically injecting a slave diode laser.

Cooling light is coupled into fibers that are connected to an integrated fiber splitter system (Schäfter\&Kirchhof) which distributes the light to the 6 MOT beams which have an intensity of $4.2 \mathrm{~mW} / \mathrm{cm}^{2}$ each with $11 \mathrm{~mm}$ beam waist. Each beam is delivered to the MOT region through a collimator rigidly fixed to the cell. Hyperfine repumping light is provided by a beam in one direction with an intensity of $0.8 \mathrm{~mW} / \mathrm{cm}^{2}$.

Under standard operating conditions, $\sim 10^{9}$ atoms are loaded in the MOT with a typical loading time $\tau=2.5 \mathrm{~s}$. The MOT has a size of $\sim 4 \mathrm{~mm}$.

The atomic cloud is launched into the fountain tube tube by using the moving molasses method. The laser beams propagating upwards and downwards are separately controlled by two acousto-optic modulators (AOMs). By applying a detuning $\delta \omega$ of opposite sign to the upper and lower beams, the atoms are forced to move along the vertical direction with a velocity

$$
v=\frac{\delta \omega}{k \cos \xi}
$$

where $k$ is the wave vector of the cooling radiation and $\xi$ the common angle between each of the six beams and the vertical direction $(\cos \xi=1 / \sqrt{3})$. During the launch, the detuning of the cooling beams in the moving frame is increased and their intensity is reduced. This sequence allows to further cool the atomic sample in the moving molasses frame. The launch sequence for one cloud is realized in four steps (Figure 2). After the MOT magnetic field is switched off, the atoms are launched upwards by introducing a detuning of opposite sign to the three upwards- and downwards-propagating laser beams. After $2.5 \mathrm{~ms}$, the intensity of the beams is lowered to the saturation intensity $\mathrm{I}_{\mathrm{s}}=1.7 \mathrm{~mW} / \mathrm{cm}^{2}$ and the mean detuning is increased to $-6.3 \Gamma$. After $1.8 \mathrm{~ms}$, the intensity of the cooling beams is reduced by a factor of 2 for $0.3 \mathrm{~ms}$. Finally, the cooling beams are switched off, leaving only the repumper beam on to optically pump the atoms into the $\mathrm{F}=2$ state. The temperature of the sample, measured by monitoring its axial expansion during free fall, is approximately $4 \mu \mathrm{K}$. The number fluctuation from launch to launch is about $3 \%$. The fountain sequence is synchronous with the $50 \mathrm{~Hz}$ power line.

The gradiometer requires two clouds of cold atoms moving with the same velocity at the same time, but vertically displaced. A vertical separation of $35 \mathrm{~cm}$ for atoms 


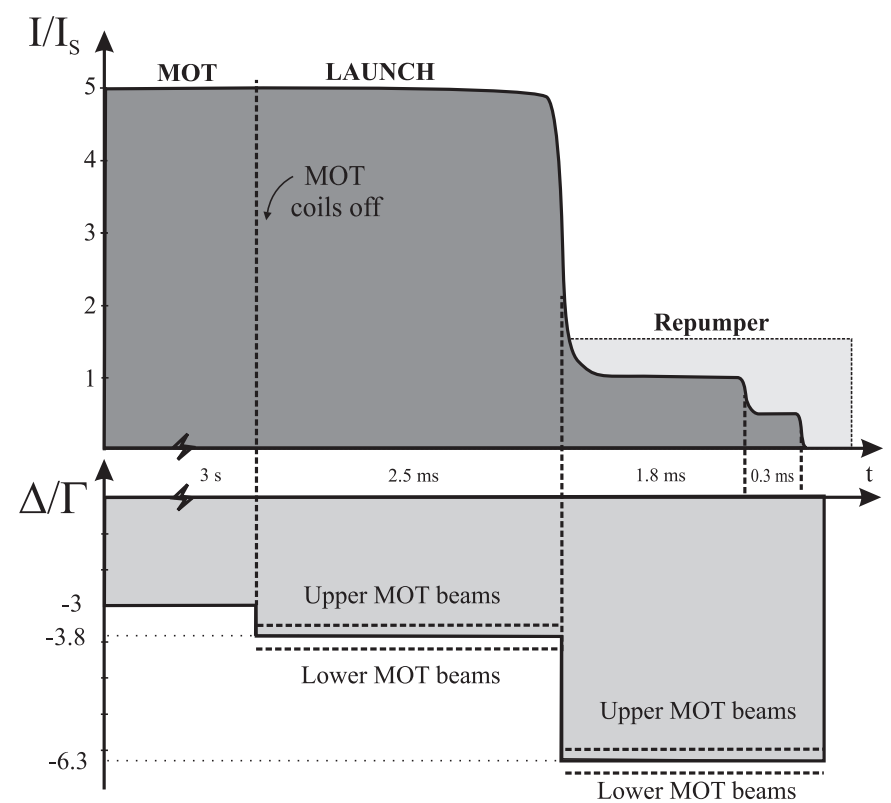

Figure 2. Sequence used in the experiment to launch cold atoms in the fountain. Horizontal dashed lines represent the different frequencies for the downwards- and upwardspropagating beams. The time axis is not to scale.

launched $60 \mathrm{~cm}$ and $95 \mathrm{~cm}$ above the MOT results in a launch delay between the two clouds of about $100 \mathrm{~ms}$. The two atomic clouds are prepared using the juggling technique [19. During the ballistic flight of the first cloud of atoms a second cloud is loaded from the backgroud vapour. Just before the first cloud falls down in the MOT region, the second one is launched. Then, the first cloud, used as a cold and intense source of atoms, is recaptured, cooled and launched upwards within less than $50 \mathrm{~ms}$ (Figure 3).

To optimize the juggling sequence, several factors must be taken into account. First, the recapture efficiency of the atomic fountain decreases with the launch height. The dominant loss process is the scattering with the thermal background atoms. The loss process due to the thermal expansion of the cloud during the flight is so far negligible. Second, the higher the launch of the first cloud, the longer the loading time for the second one. Third, increasing the time used to collect atoms for the first cloud has two opposite effects: the number of recaptured atoms after the launch is higher, but the experimental repetition rate is reduced, and so the instrument sensitivity.

In our experimental sequence, the first cloud is launched $60 \mathrm{~cm}$ upwards, which leads to a loading time of $650 \mathrm{~ms}$ for the second cloud. In this way the number of atoms launched in each of the two clouds used in the gradiometer is $5 \cdot 10^{8}$.

\subsection{State preparation procedure}

After the launch, the atoms are selected both in velocity and by their $m_{F}$ state. The selection procedure uses vertical beams so that the state preparation can take place

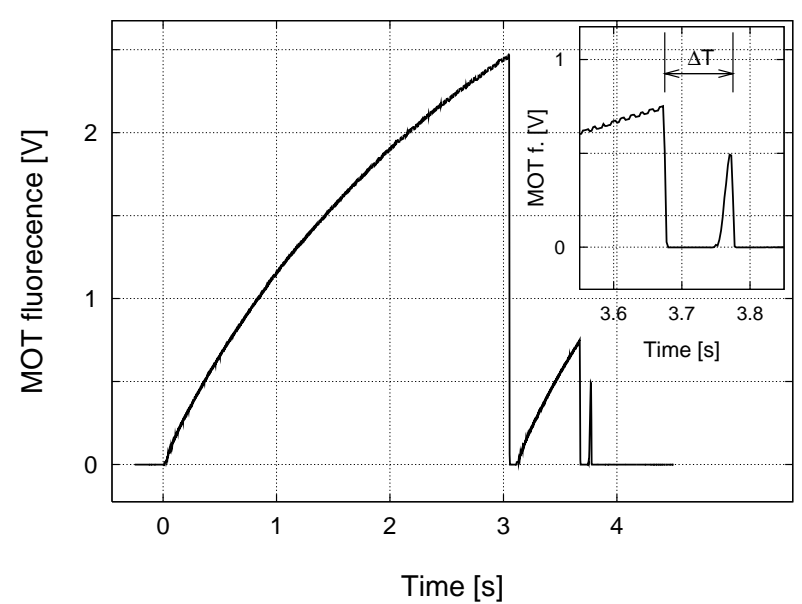

Figure 3. MOT Fluorescence detected during a juggling sequence. The first two clouds are slowly loaded from background gas. The third one is obtained trapping the first cloud falling down in the MOT region. The inset shows the launch of the last two clouds in a time interval of $100 \mathrm{~ms}$.

simultaneously on both clouds. The selection is realized in the vacuum tube, where a uniform vertical bias field of $25 \mu \mathrm{T}$ is applied. The sequence starts by blowing away the residual population in the $\mathrm{F}=1$ state, by applying a $3 \mathrm{~ms}$ pulse of elliptically polarized radiation resonant with the $\mathrm{F}=1 \rightarrow \mathrm{F}^{\prime}=0$ transition. This beam is tilted with respect to the vertical direction so that $\Delta \mathrm{m}_{\mathrm{F}}=0, \pm 1$ are possible. A narrow selection of the vertical velocity distribution of atoms in the $\mathrm{F}=2, \mathrm{~m}_{\mathrm{F}}=0$ is transferred to the $\mathrm{F}=1, \mathrm{~m}_{\mathrm{F}}=0$ state using a $100 \mu$ s velocity-selective Raman pulse. The atoms in the $\mathrm{F}=2$ state are removed from the sample by applying a circularly polarized vertical beam for $5 \mathrm{~ms}$, resonant with the $\mathrm{F}=2 \rightarrow \mathrm{F}^{\prime}=3$ transition.

After the selection sequence, the atoms end up in the $\mathrm{F}=1, \mathrm{~m}_{\mathrm{F}}=0$ state with a horizontal temperature of $4 \mu \mathrm{K}$ and a vertical temperature of $40 \mathrm{nK}$, corresponding to velocity distribution widths (HWHM) respectively of $3.3 v_{\text {rec }}$ and $0.3 v_{\text {rec }}\left(v_{\text {rec }}=6 \mathrm{~mm} / \mathrm{s}\right.$ for $\mathrm{Rb}$ resonance transition $)$.

\subsection{Raman interferometer apparatus}

Stimulated Raman transitions are driven by light from two extended-cavity phase-locked diode lasers, with a relative frequency difference equal to the ${ }^{87} \mathrm{Rb}$ ground state hyperfine splitting frequency $\left(\nu_{\mathrm{hf}}=6.835 \mathrm{GHz}\right)$ and amplified by a single tapered amplifier. The detuning from the $\mathrm{D}_{2}$ resonance is $-3.3 \mathrm{GHz}$.

The laser locking system was described in detail in [20]. The two main requirements of the optical phase-lockedloop (OPLL) are robustness, necessary for continuous operation over long periods of time, and low rms phase error $\sqrt{\left\langle\varphi^{2}\right\rangle}$, a limiting parameter for the interferometer sensitivity. These requirements are accomplished using a detector that combines in a mutually exclusive way a digital phase and frequency detector and an analog phase detector. The digital detector allows a capture range of the 
order of $100 \mathrm{MHz}$, whereas the analog detector ensures a low noise spectral density necessary for accurate phaselocking.

The master laser (ML) is an extended-cavity diode laser (New Focus Vortex 6000, $65 \mathrm{~mW}$ at $130 \mathrm{~mA}$ ). The slave laser (SL) is an anti-reflection coated diode (Sacher SAL-0780-040, $35 \mathrm{~mW}$ at $60 \mathrm{~mA}$ ) mounted in an extended cavity. The ML and SL beams are combined on a polarizing beam splitter (PBS) followed by a linear polarizer. One output of the PBS is sent to a fast photodiode to generate the beat note. The second PBS output injectionseeds a tapered amplifier, whose astigmatism is corrected for with a cylindrical lens. A hot Rb cell filters resonant light in the spectral wings of the amplifier output.

The pulse timing of the Raman beams during the interferometer sequence is controlled by an AOM, driven by a waveform generator (Agilent 33220A). After the AOM, the beams are coupled into a polarization-maintaining fiber, which acts as a spatial filter. The fiber output is collimated by an aspherical lens $(\mathrm{f}=1000 \mathrm{~mm})$ to obtain a beam waist of $10 \mathrm{~mm}$. Most of the optical elements on the path of the Raman beams have a $(\lambda / 20)$ quality to avoid phase-front distorsions.

The Raman beams enter the vacuum system through the lower window of the MOT cell and exit through the window at the top of the interferometer tube. After passing through a quarter-wave plate, they are retroreflected by a mirror, thus obtaining a lin $\perp$ lin configuration in the interferometer region. The horizontality of the retroreflecting mirror has been adjusted within $20 \mu$ rad using a tiltmeter (Applied Geomechanics 755-1129). Taking into account the Doppler effect and considering the polarization of the beams, only Raman transitions with $\Delta \mathrm{m}_{\mathrm{F}}=0$ are possible for atoms with non zero velocity. To compensate for the varying Doppler shift of the atomic resonance during the atoms free-fall trajectory, the Raman beams frequency difference is linearly swept using a continuousphase waveform generator (Agilent 33250A). The central $\pi$ pulse of the interferometer sequence is sent $5 \mathrm{~ms}$ before the atoms reach the top of their trajectory, when their velocity is still high enough to discriminate between upwards and downwards propagating Raman beams. For a Raman beam intensity of $30 \mathrm{~mW} / \mathrm{cm}^{2}$, the $\pi$ pulse lasts $100 \mu \mathrm{s}$.

The interferometric phase shifts are detected using the relative phase of the Raman beams as a reference. To scan the interferometric fringes, a controlled phase jump $\phi_{\mathrm{L}}$ is applied after the $\pi$ pulse to the rf signal generated by the low-phase-noise reference oscillator (Anritsu MG3692A).

Coils wound between the vacuum tube and the magnetic shield enable a precise control of the magnetic field during the interferometer sequence. A solenoid, of $1 \mathrm{~m}$ length, generates a vertical bias magnetic field on the $z-$ axis, chosen as quantization direction in the interferometric region. A series of 10 coils, each $9 \mathrm{~cm}$ long, are used to add a controlled phase shift via the second-order Zeeman shift on the atoms during the interferometer sequence.

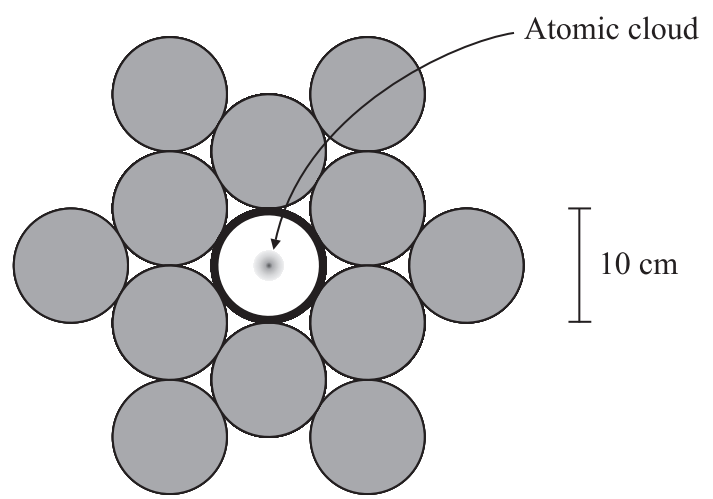

Figure 4. Source masses position around the interferometer tube (top view).

\subsection{Detection scheme}

After the interferometric sequence, the population of the two hyperfine sublevels of the ground state is measured in the intermediate chamber using normalized fluorescence detection. The falling clouds pass through two horizontal counterpropagating circularly-polarized beams, vertically displaced by $20 \mathrm{~mm}$. The beams have a rectangular section of $(13 \times 6) \mathrm{mm}^{2}$, and their wave vector $\boldsymbol{k}$ is parallel to a local magnetic field. Each beam has a power of $1 \mathrm{~mW}$ and is resonant with the $\mathrm{F}=2 \leftrightarrow \mathrm{F}^{\prime}=3$ transition, in order to interact with atoms in the $\mathrm{F}=2$ state. The lower part of the higher beam is not retroreflected in order to horizontally blow-away the atoms after detection. On the lower probe beam $0.4 \mathrm{~mW}$ of repumping light is overlapped to optically pump atoms from the $\mathrm{F}=1$ state to the closed $\mathrm{F}=2 \leftrightarrow \mathrm{F}^{\prime}=3$ transition. With this configuration, atoms in the $\mathrm{F}=2$ state are detected with the higher beam and atoms in the $\mathrm{F}=1$ state with the lower one. The fluorescence photons from the two detection regions are collected by a $5 \mathrm{~cm}$ diameter lens $(\mathrm{f}=100 \mathrm{~mm})$ placed at a distance of $130 \mathrm{~mm}$ and separately focused onto two large area photodiodes (Hamamatsu S7539). The population fractions in the two states are obtained via normalization, eliminating the shot-to-shot dependence on atom number. With a typical number of $5 \cdot 10^{4}$ detected atoms per cloud in each state, the signal-to-noise ratio is $60 / 1$, limited by background light.

\subsection{Source masses set-up}

Two sets of masses are used to generate a well-known gravitational field. Each set is made of 12 identical cylinders, symmetrically arranged in a hexagonal configuration around the vertical axis of the atomic fountain (Figure 4). The cylinders have a diameter of $100 \mathrm{~mm}$ and a height of $150 \mathrm{~mm}$. The two sets of masses are held by two titanium rings, which are connected to a precision translation stage specifically designed for the experiment. This allows to move vertically the two sets of masses and to position them at a relative distance ranging between 4 and $50 \mathrm{~cm}$. The distance is measured with two linear optical encoders 
(Heidenhain LS603). A test of the system with a lasertracker showed a reproducibility of $\pm 1 \mu \mathrm{m}$.

In the final configuration for the $G$ measurement, wellcharacterized W masses will be used (see Section 6). The results reported in this paper were instead obtained using $\mathrm{Pb}$ source masses. Each cylinder had a mass of $12.80 \mathrm{~kg}$. The total mass of the 24 cylinders was $307.2 \mathrm{~kg}$.

\section{Atom interferometer operation and characterization}

In the gravimeter configuration, the atom interferometer operating with a single sample of atoms launched in the fountain, the main phase term is the one induced by Earth's gravity

$$
\phi(g)=\mathrm{k}_{\mathrm{eff}} g \mathrm{~T}^{2}
$$

where $\hbar \mathrm{k}_{\text {eff }}$ is the momentum transferred to the atoms by a Raman pulse.

The observed interference signal is shown in Figure [5] The fraction of atoms in the $\mathrm{F}=2$ state is plotted as a function of the phase $\phi_{\mathrm{L}}$, which is electronically added to the Raman lasers before the final $\pi / 2$ pulse. The total phase is in this case the sum of $\phi(g)$ and $\phi_{\mathrm{L}}$. Each data point results from a single launch of an atomic cloud $80 \mathrm{~cm}$ above the center of the MOT and requires $5.5 \mathrm{~s}$. The phase step is $10^{\circ}$. The phase change due to the varying Doppler effect during the atoms flight was cancelled by chirping the lasers' frequency. Figure 5 compares the signals recorded without vibration isolation for the mirror retroreflecting the Raman beams (except for the table air legs with active position stabilization, Newport RS 2000) and the signal recorded when an active vibration isolation system (HWL Scientific Instruments AVI 350-M(L)) was used to stabilize the mirror. The signal-to-noise ratio, defined as the ratio between twice the fringe amplitude and the rms noise, is 7 in the first case and 18 in the second case. The pulse spacing $\mathrm{T}$ can be increased up to $150 \mathrm{~ms}$, but the phase noise induced by mirror vibrations drastically reduces the fringe visibility. A good phase definition for the fringes requires a better isolation system for the mirror and for the table where the atomic fountain is mounted. A gravity gradient measurement is obtained by two vertically separated acceleration measurements. The simultaneous realization of these measurements overcomes the stringent limit set by phase noise thanks to commonmode noise rejection. The Raman sequence interval $\mathrm{T}$, as well as the gradiometer sensitivity, can then be increased up to the limit set by experimental constraints. If $g_{\text {low }}$ and $g_{\text {up }}$ are the gravity acceleration values at the position of the lower and upper interferometers, the differential phase shift is

$$
\phi(\Delta g)=\mathrm{k}_{\text {eff }}\left(g_{\text {low }}-g_{\text {up }}\right) \mathrm{T}^{2}
$$

As discussed above, the gradiometer requires two clouds of cold atoms moving with the same velocity at the same time, but vertically displaced. A vertical separation of $35 \mathrm{~cm}$ for atoms launched $60 \mathrm{~cm}$ and $95 \mathrm{~cm}$ above the MOT requires a launch delay between the two clouds of

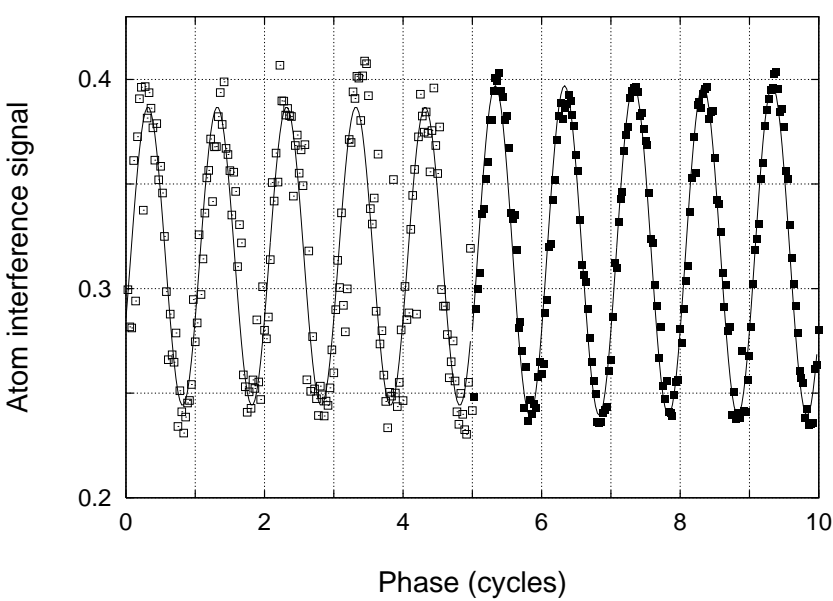

Figure 5. Fringes recorded with a single atom interferometer for $\mathrm{T}=20 \mathrm{~ms}$ delay between Raman pulses. The active vibration isolation system of the mirror was off during the first five cycles (empty squares) and on during the last five (filled squares). The acquisition time for each data set ( 5 cycles) was $900 \mathrm{~s}$. The solid lines are sinusoidal least-square fits over the two sets of data. The phase uncertainty is $33 \mathrm{mrad}$ in the first case and $10 \mathrm{mrad}$ in the second case.
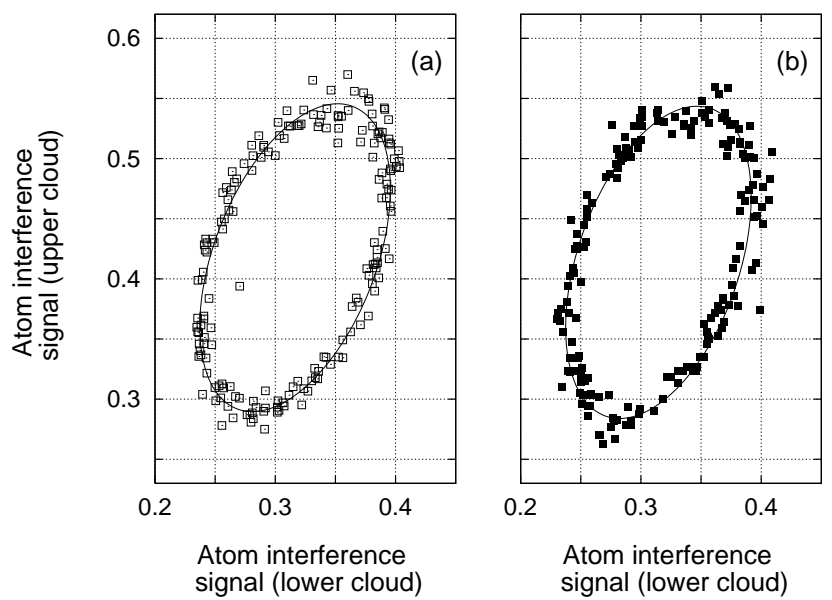

Figure 6. Signal from the gradiometer with $\mathrm{T}=20 \mathrm{~ms}$. The fringes from the upper gravimeter are plotted versus the fringes from the lower interferometer. The relative phase can be extracted from the elliptic fit parameters. The mirror isolation system was on in (a), off in (b). The error on the relative phase is $\sim 10 \mathrm{mrad}$.

about $100 \mathrm{~ms}$. In the present apparatus, the two atomic clouds are prepared using the juggling technique. In the final configuration, a 2D-MOT will be used for faster loading of the MOT [18.

The Earth's gravity gradient $\left(\sim 3 \cdot 10^{-6} \mathrm{~s}^{-2}\right)$ corresponds to a relative phase shift that, for a vertical distance $\Delta z$ of $35 \mathrm{~cm}$ and a time interval $\mathrm{T}=100 \mathrm{~ms}$ between the Raman pulses, is expected to be 175 mrad. A sensitive measurement of the relative phase of the two interferometers is obtained by using an ellipse fitting method 21] to cancel common-mode phase-noise. The interference signal of the upper interferometer is plotted versus that of 


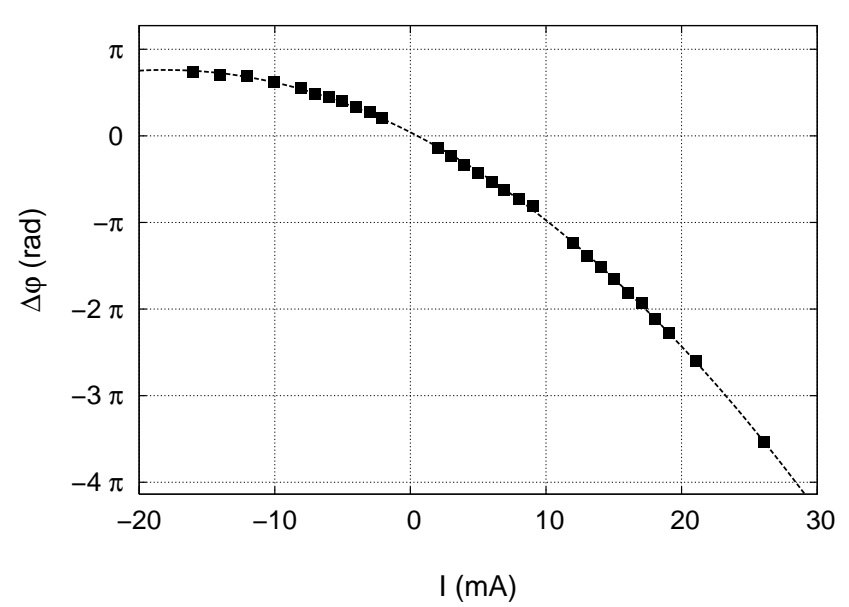

Figure 7. Phase shift induced by applying a magnetic field pulse with different amplitudes to the lower interferometer for $\mathrm{T}=100$ ms delay between Raman pulses.

the lower (Figure [6). The data then describe an ellipse and the relative phase shift can be obtained from its eccentricity and rotation angle. The measured signal-to-noise ratio is about 100/1 limited by residual amplitude noise.

To adjust the operating point of the gradiometer, a controlled phase shift was added to the lower interferometer using a magnetic field pulse, acting on the $\mathrm{m}_{\mathrm{F}}=0$ atoms via the second-order Zeeman effect. The resulting phase shift can be written as

$$
\Delta \phi_{B}=2 \pi \nu_{\mathrm{z}, \mathrm{II}}\left(B^{2}\right) \int_{0}^{t_{m}}\left(B_{\text {low }}^{2}(t)-B_{\text {up }}^{2}(t)\right) \mathrm{dt}
$$

where $\nu_{\mathrm{z}, \mathrm{II}}\left(B^{2}\right)=57.515 \mathrm{GHz} / \mathrm{T}^{2}$ is the second order Zeeman shift coefficient for ${ }^{87} \mathrm{Rb}, t_{m}$ is the duration of the magnetic pulse, $B_{\text {low }}$ and $B_{\text {up }}$ the magnetic field values at the lower and upper accelerometer, respectively.

In this case, a knowledge of the pulsed magnetic field is needed for a precise gradient measurement, although it is not critical in the double-differential scheme for the $G$ measurement where only stability matters. The magnetic field in the interferometric region was mapped for different current values in the bias coils using atoms in different $\mathrm{m}_{\mathrm{F}}$ sublevels as probes. A sample of cold atoms in the $\mathrm{F}=2$ state was repeatedly launched in the interferometer tube and a selected velocity class was transferred to the $\mathrm{F}=1$ state with a Raman pulse before reaching the inversion point. The atomic sample was then transferred back to the $\mathrm{F}=2$ state with a $\pi$ Raman pulse and selectively detected. The delay of the second pulse was varied, in order to interact with the free-falling atoms at different vertical positions. This measurements also provide the optimal value for the Raman beams frequency ramp, which is $25.1354(3) \mathrm{MHz} / \mathrm{s}$ corresponding to a gravity acceleration $g$ of $9.8056(1) \mathrm{m} / \mathrm{s}^{2}$. In Figure [7] a direct measurement of the magnetically-induced phase shift is shown. Each point results from an elliptic fit on a set of gradiometric measurements for a certain current value in a bias coil. The parabolic fit does not cross the axis origin but is shifted by $125 \mathrm{mrad}$. This vertical offset shoud be induced by the Earth's gradient that is constantly present during the whole set of measurements. This result differs from the value of $175 \mathrm{mrad}$ produced by the Earth's gravity gradient. The difference can be attributed to the Coriolis effect.

For an atomic velocity component $\mathbf{v}_{E W}$ along EastWest direction, the phase induced by the Coriolis effect is

$$
\Delta \phi_{C}=2 \boldsymbol{\Omega} \cdot\left(\mathbf{v}_{\mathrm{EW}} \times \mathbf{k}_{\mathrm{eff}}\right) \mathrm{T}^{2}
$$

where $\boldsymbol{\Omega}$ is the Earth's rotation frequency $\left(\boldsymbol{\Omega}=7.29 \times 10^{-5}\right.$ $\mathrm{rad} / \mathrm{s}$ ). At the lab location (latitude $\theta_{\text {lat }}=43^{\circ} 47^{\prime}$ ), a $\mathbf{v}_{\mathrm{EW}}$ component of $1 \mathrm{~cm} / \mathrm{s}$ introduces a $\Delta \phi_{C}$ corresponding to an acceleration $\Delta a=2 \boldsymbol{\Omega} \mathbf{v}_{\mathrm{EW}} \cos \theta_{\text {lat }}=10^{-7} \mathrm{~g}$.

In the same conditions, the resulting signal for the gradiometer configuration is proportional to the horizontal velocity difference between the two atomic samples:

$$
\Delta \phi_{C}=2 \boldsymbol{\Omega} \cdot\left(\boldsymbol{\Delta} \mathbf{v}_{\mathrm{EW}} \times \mathbf{k}_{\mathrm{eff}}\right) \mathrm{T}^{2}
$$

For launch heights of 60 and $95 \mathrm{~cm}$ above the MOT, the resulting phase difference corresponds to $2 \cdot 10^{-8} \mathrm{~g}$. This effect can be minimized by a velocity selection of the atoms launched in the interferometer [22].

For the determination of $G$, the gradiometer signal will be detected as a function of the source masses positions so that rotational contributions cancel and only fluctuations of the launch direction and height within the measurement time can affect the signal.

\section{Detection of source masses}

In order to test the apparatus and to show the possibility of detecting the effect of external source masses, the interferometer was operated in gradiometer configuration and a set of $\mathrm{Pb}$ masses was used.

$\mathrm{Pb}$ cylinders had the shape and arrangement as described in Section 3.5 Masses were alternately set in the positions corresponding to the configuration (1) and the configuration (2) shown in Figure 1 In the first case, the acceleration induced on the atoms in the upper cloud is in the $-z$ direction and the acceleration for the lower cloud is in the $+z$ direction. The sign of the induced acceleration is changed, moving the masses to the configuration (2) with respect to the atomic clouds.

In Figure 8 the differential phase shifts measured for the two sets of $\mathrm{Pb}$ cylinders in the two configurations are reported. Considering the differences between two consecutive measurements, the resulting phase shift from the whole data set is $144(5) \mathrm{mrad}$, which corresponds to a sensitivity of $3 \cdot 10^{-9} \mathrm{~g}$ and a relative uncertainty of $4 \cdot 10^{-2}$ in the measurement of $G$. The total data acquisition time was about $5 \mathrm{~h}$.

By evaluating the difference of consecutive measurements, a reduction of systematic effects, due for instance to spatially inhomogeneous spurious accelerations, Earth's gravity gradient, inhomogeneous electric and magnetic fields and inertial forces, is achieved. 


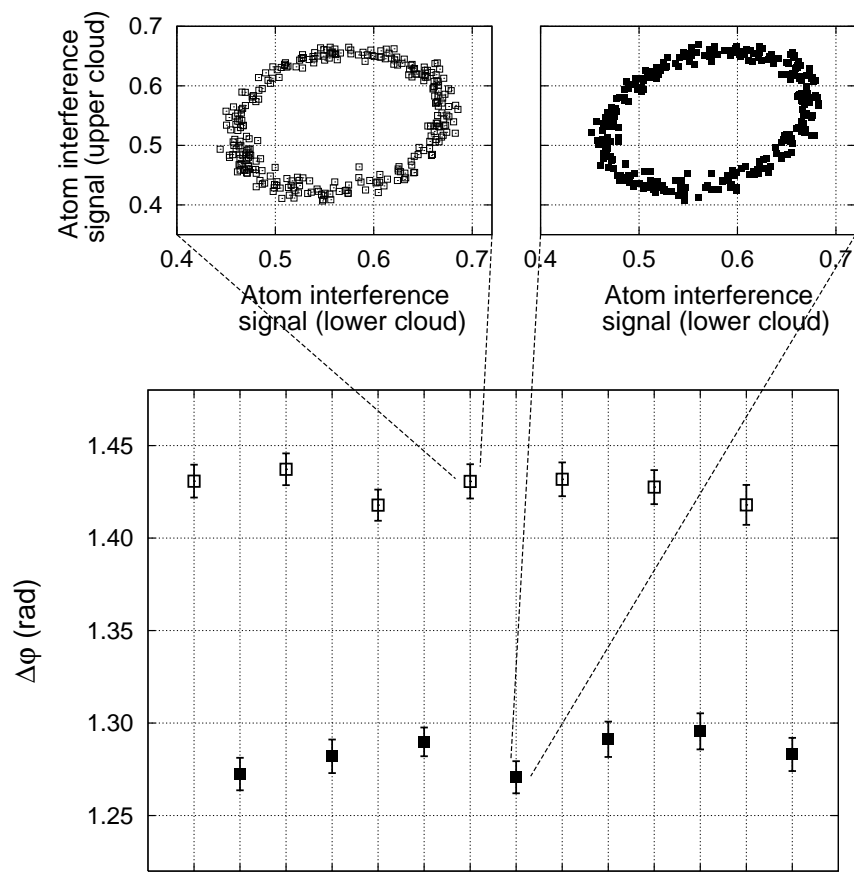

Figure 8. Gravitational phase shift detected for the two positions of the source masses. The experiment was performed with $\mathrm{Pb}$ masses and data were analyzed using the ellipse fitting method. Each data point results from a fit over 288 sequences with a phase step of $5^{\circ}$ for the local oscillator. The acquisition time for each point is 20 minutes. The translation of the source masses between two consecutive measurements required $\sim 100 \mathrm{~s}$. The full data set for two measurements in different configurations are also shown in the upper part of the figure.

\section{Towards the measurement of $\mathrm{G}$}

To reach the planned accuracy of $\Delta G / G=10^{-4}$ in the measurement of $G$, an optimization and characterization of the interferometer and source mass apparatus are required.

The source masses material for the $G$ measurement is a sintered alloy made of tungsten $(95.3 \%)$, nickel $(3.2 \%)$ and copper $(1.5 \%)$ (Plansee INERMET IT180). This material is non-magnetic and has a typical density of (18000 \pm 200) $\mathrm{kg} / \mathrm{m}^{3}$. The 24 cylinders for the experiment come out of the same furnace run. Then the cylinders undergo a hot isostatic pressing (HIP) treatment at $\mathrm{T}=1200^{\circ} \mathrm{C}$, $\mathrm{P}=1000$ bar which improves the homogeneity of the material.

Characterization tests on this material were performed before and after the HIP treatment. Samples were observed by a microscope to detect internal holes; holes, with a typical diameter of $\sim 150 \mu \mathrm{m}$, were reduced to a negligible size by the HIP treatment. The material mean density was measured through a double weighting (in air and in water) at constant temperature. An ultrasonic test was performed to study its homogeneity that also showed a significative improvement after the HIP procedure. We measured the dimensions and deviations from the cylin-

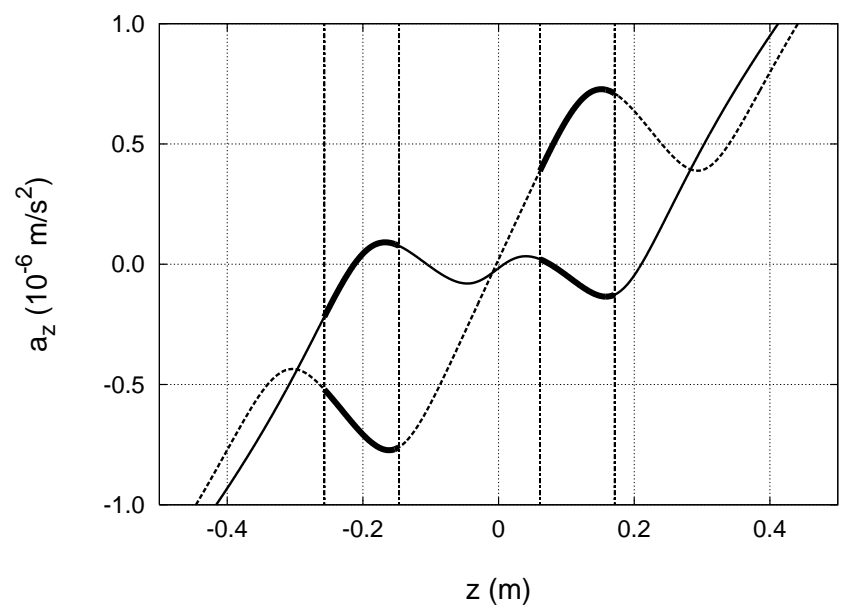

Figure 9. Vertical acceleration computed for the final experiment with tungsten source masses in two positions. The Earth's gravity gradient was considered. The solid line corresponds to configuration 1 and the dashed line to configuration 2. The spatial regions corresponding to the atomic trajectories for the two interferometers are highlighted.

dric shape and verified that they can be machined with a precision of 1-2 $\mu \mathrm{m}$. A sample cylinder was cut into blocks of $(25 \times 25 \times 44) \mathrm{mm}^{3}$ to characterize the internal density distribution. The results of all tests show that masses should not affect the final precision planned for the $G$ measurement.

The parameters of the atom interferometer must be optimized considering the shape of the gravitational field produced by the source masses. For the configuration 1 in Figure 9 selected atomic trajectories will maximize the phase difference between the two interferometers. Keeping the same atomic trajectories, the source masses will be moved to configuration 2 in which the phase difference term has minimum sensitivity to the atomic position. In this way, the interferometers will be realized where the acceleration is stationary for both configurations. $\Delta G / G=10^{-4}$ can be reached assuming for the atoms a uncertainty of 1 $\mathrm{mm}$ for the position and $5 \mathrm{~mm} / \mathrm{s}$ for the velocity. Higher levels of precision and accuracy are imposed on the knowledge of the distance between the source masses $(10 \mu \mathrm{m})$ and of the relative positioning $(100 \mu \mathrm{m})$. This will be achieved by a combination of stable mechanical positioning and translation mount and direct optical measurement of the position of the cylinders during the experiment.

\section{Conclusions}

The apparatus developed to measure $G$ using a new scheme based on atom interferometry was presented. The atom interferometer is used to measure the gravitational field produced by source masses. A differential scheme, realized by an atom juggling procedure, was implemented working as a gravity-gradiometer, thus reducing commonmode noise. The result of a preliminary experiment was reported, where the effect of $\mathrm{Pb}$ source masses was clearly 
A. Bertoldi et al.: Atom interferometry gravity-gradiometer for the determination of the Newtonian...

detected. Further improvements in the Rb atomic fountain, a reduction of noise sources and the use of well characterized $\mathrm{W}$ source masses will allow to reach the projected precision in the measurement of $G$.

This work was supported by INFN (MAGIA experiment), MIUR, ESA, and EU (under contract RII3-CT-2003-506350). G.M.T. acknowledges seminal discussions with M. A. Kasevich and J. Faller. We are grateful to B. Dulach of INFN-LNF for the design of the support for the source masses and to A. Peuto of INRIM for density tests on $\mathrm{W}$ masses.

\section{References}

1. P.R. Berman, ed., Atom interferometry (Academic press, 1997)

2. A. Peters, K.Y. Chung, S. Chu, Nature (London) 400, 849 (1999)

3. M.J. Snadden, J.M. McGuirk, P. Bouyer, K.G. Haritos, M.A. Kasevich, Phys. Rev. Lett. 81, 971 (1998)

4. S. Fray, C.A. Diez, T.W. Hänsch, M. Weitz, Phys. Rev. Lett. 93, 240404 (2004)

5. G. M. Tino, High precision gravity measurements by atom interferometry. In A Relativistic Spacetime Odyssey - Proceedings of JH Workshop, Firenze, 2001 (World Scientific, 2003). Also, G. M. Tino, Nucl. Phys. B (Proc. Suppl.) 113, 289 (2003).

6. S. Dimopoulos, A.A. Geraci, Phys. Rev. D 68, 124021 (2003)

7. G. Ferrari, N. Poli, F. Sorrentino, G.M. Tino, arXiv:physics/0605018 (2006, to be published)

8. J.M. McGuirk, G.T. Foster, J.B. Fixler, M.J. Snadden, M.A. Kasevich, Phys. Rev. A 65, 033608 (2002)

9. T.L. Gustavson, P. Bouyer, M.A. Kasevich, Phys. Rev. Lett. 78, 2046 (1997)

10. F. Yver-Leduc, P. Cheinet, J. Fils, A. Clairon, N. Dimarcq, D. Holleville, P. Bouyer, A. Landragin, J. Opt. B: Quantum Semiclass. Opt. 5, S136 (2003)

11. HYPER, Hyper Precision Cold Atom Interferometry in Space, Assessment study report, ESA-SCI (2000) 10

12. C. Bordé, G.M. Tino, F. Vetrano, 2004 Aspen Winter College on Gravitational Waves (Available online at: http://www.ligo.caltech.edu/LIGO_web/Aspen2004/pdf/ vetrano.pdf, to be published)

13. R.Y. Chiao, A.D. Speliotopoulos, Journal of Modern Optics 51(6-7), 861 (2004)

14. A. Roura, D.R. Brill, B.L. Hu, C.W. Misner, W.D. Phillips, Phys. Rev. D 73, 084018/1 (2006)

15. M. Fattori, G. Lamporesi, T. Petelski, J. Stuhler, G.M. Tino, Phys. Lett. A 318, 184 (2003)

16. P.J. Mohr, B.N. Taylor, Rev. Mod. Phys. 77-1, 42 (2005)

17. M. Kasevich, S. Chu, Appl. Phys. B 54, 321 (1992)

18. T. Petelski, Atom interferometers for precision gravity measurements, $\mathrm{PhD}$ Thesis, Università di Firenze (2005). Available online at www.fi.infn.it/sezione/esperimenti/MAGIA/home.html

19. R. Legere, K. Gibble, Phys. Rev. Lett. 81, 5780 (1998)

20. L. Cacciapuoti, M. de Angelis, M. Fattori, G. Lamporesi, T. Petelski, M. Prevedelli, J. Stuhler, G.M. Tino, Rev. Sci. Instrum. 76, 053111 (2005)
21. G.T. Foster, J.B. Fixler, J.M. McGuirk, M.A. Kasevich, Opt. Lett. 27, 951 (2002)

22. A. Peters, K.Y. Chung, S. Chu, Metrologia 38, 25 (2001) 